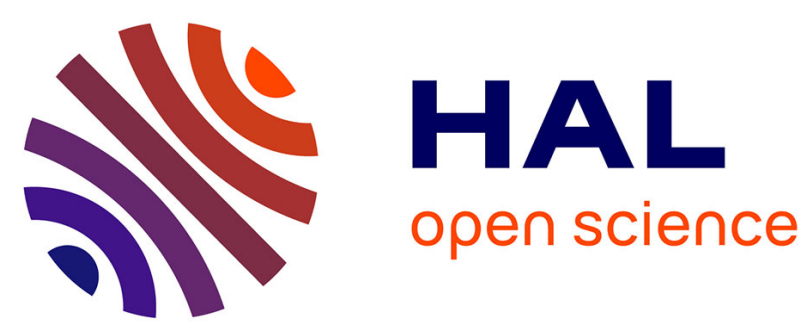

\title{
Enduits peints d'Orléans
}

Claudine Allag

\section{To cite this version:}

Claudine Allag. Enduits peints d'Orléans. Gallia - Fouilles et monuments archéologiques en France métropolitaine, 1983, 41 (1), pp.191-200. 10.3406/galia.1983.1887 . hal-01940251

\section{HAL Id: hal-01940251 \\ https://hal.science/hal-01940251}

Submitted on 27 Feb 2020

HAL is a multi-disciplinary open access archive for the deposit and dissemination of scientific research documents, whether they are published or not. The documents may come from teaching and research institutions in France or abroad, or from public or private research centers.
L'archive ouverte pluridisciplinaire HAL, est destinée au dépôt et à la diffusion de documents scientifiques de niveau recherche, publiés ou non, émanant des établissements d'enseignement et de recherche français ou étrangers, des laboratoires publics ou privés.

\section{(이)(\$)}

Distributed under a Creative Commons Attribution - NonCommercial - NoDerivatives| 4.0 


\title{
NOTES \\ ENDUITS PEINTS D'ORLÉANS
}

\author{
par Claudine ALLAG
}

Ine dizaine de caisses de fragments de peinture murale nous ont ale ronfices par II. Y. De Kisch. Ces peintures proviennenl d'une salle thermale (quartier llavien); elles etaient entassées sur le sol d'une pelite niche reclangulaire (de $2 \times 1,20 \mathrm{~m}$ env.), sur le roite surl de celte salle.

Les fragments ont éte nelloyés, enduits de fixatif, et assemblés loules les fois que cela étail possible. Ies cind principaux ensembles de morceaux recollés ont éte inclus dans un mortier moderne, alin de pouvoir elre facilement manipules el présentés. Cing panneaux ont été ansi constitues, dont les dimensions sont les suivantes : $51 \times 10 \mathrm{~cm}, 5) 1 \times 50(\mathrm{~cm}$. $31 \times 26 \mathrm{~cm}, 42 \times 26 \mathrm{~cm}, 29 \times 39 \mathrm{~cm}$.

Ces peintures représentent un ensemble parfaitement homogine. aussi bien par la nature du support que par l'aspect de la surface décorative. Le remblai na certainement pas été remanié, et il s'agit selon loute vraisemblance d'un décor provenant de la niche mème dans laquelle il a éte trouvé La surface de l'enduit, d'ailleurs, est tres legorement concave. Le plan de la niche al anl rectangulaire et non arrondi, la peinture ne peut pass provenir des murs : il faut alors supposer qu'elle étail rouverte d'une voùte en berceau, et que c'est, le revètement de celte voùte qui est tombé a terre. Le style du décor, Ires simple, constitué d'un réseau régulier de cercles ef de points, encadré de bandes colorées, est par ailleurs caracteristique d'un plafond.

\section{La méparalion du support.}

L'étude du revers et de la tranche des fragments permet de relrouver les dilférentes élapes: de la réalisation de ce plafond.

Au revers, les empreintes bien reconnaissables sont celles de tubuli en terre cuite. rectangulaires, aux dimensions approximatives de $12,18 \mathrm{~cm}$. Ils sont marqués de deux traits diagonaux, en rreux, dans lesquels s'insere le mortier qui les revol, et qui en garde la trace. Les lubulures sont disposées en lignes paralleles a l'axe de la voute. La courbure du berceau est donc donnée par une succession de pans coupés d'une largeur égale à celle des tubulures.

L'effondrement, de ce support a entraîné des fragments d'enduit présentant, pour la plupart, les dimensions d'un lubulus.

C'est une premiere couche de mortier qui a permis de régulariser la voute et de transformer en courbe la surface morcelée. Son épaisseur varie par conséquent de 18 a 25) mm. Elle est constituée d'un mortier rose homogine, tris dur, fait de sable, de chaux, de paille et de tuileau dont quelques gros élément.s sont bien apparents (fig. 1). Par-dessus, une couche de transition a elé étendue, épaisse de $17 \mathrm{~mm}$. 


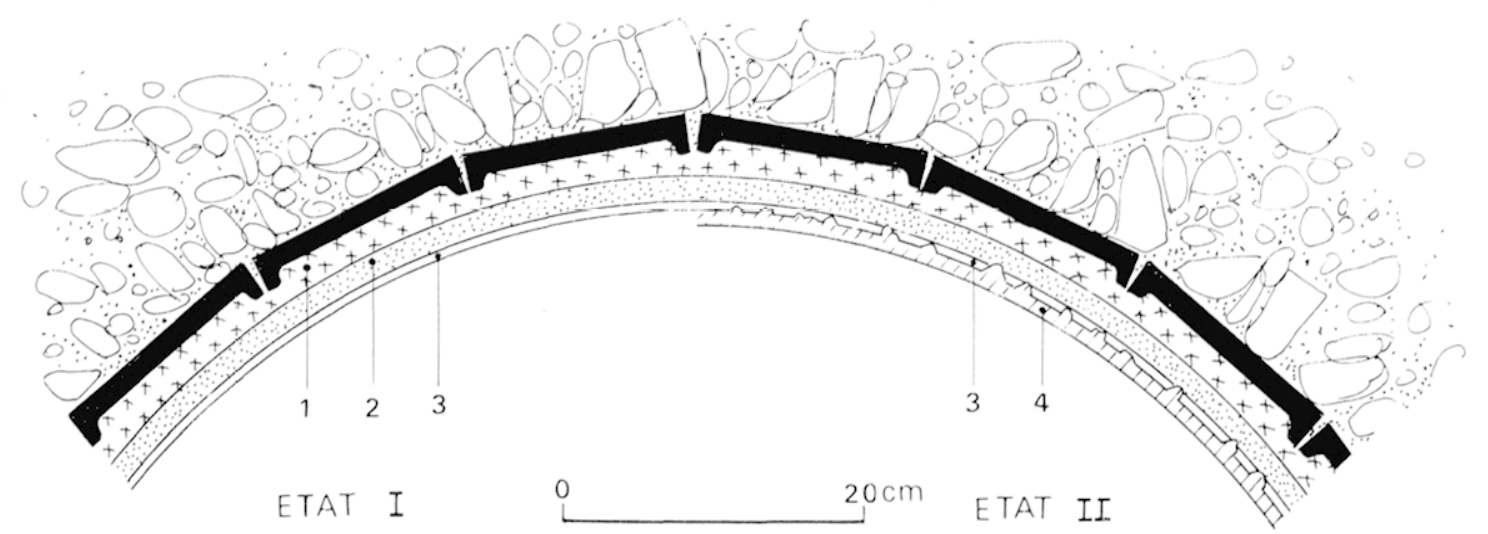

1 doupe de l'enduit. Elat $I: 1$, couche de preparation chaux el sable, luileau, paille, épaisseur : 17 a $25 \mathrm{~mm}$; 2 , couche de lransition chaux et sable, graviers ronds, epaisseur : $17 \mathrm{~mm} ; 3$, couche de surface chaux et sable fin. épaisserur : $3 \mathrm{~mm}$. Elal $I I: 3$, surface piqueté; 4 , chaux et sable fin, épaisseur : $8 \mathrm{~mm}$.

blanche, en chaux chargée de sable et de pelits graviers ronds. Enfin, la couche de surface, support direct a la peinture, est plus fine (:3 mm), et comporle seulement de la chaux el du sable tres fin. Le lait de chaux final est passé à la brosse.

\section{L'esquisse du decor.}

Cne esquisse assez soignée a précédé l'exéculion du décor. C'est une trame géométrique fondée sur un double quadrillage, et des cercles (fiq. 2). I es lignes ont été tracées avant le séchacre de l'enduit a l'aide d'une pointe asse\% émoussée qui laisse des traces plutòt larges, entaillant peu le support. Le premier Iracé consiste en un réseau de lignes perpendiculaires formant des carrés de $14,5 \mathrm{~cm}$ de còté. Ensuile. les diagonales des carrés constituent un second quadrillage, oblique par conséquent par rapport au précédent, de $10 \mathrm{~cm}$ de colé. Des décalages se produisent évidemment aux intersections ; les angles ne se superposent pas toujours. Cette hase tres simple a permis d'établir régulièrement le décor.

Le decor.

Chaque intersection du premier réseau est. marquée d'un rond peint en noir $(42 \mathrm{~mm}$ de diamètre), entouré d'un cercle bleu (diamètre lotal $70 \mathrm{~mm}$ ). I e trou de la pointe fixe d'un compas est bien visible au centre de chaque cercle, mais il n'existe aucune incision circulaire. Il faut donc supposer que ces motifs ont élé délimités à l'aide d'un compas sans double pointe siche, mais a pinceau traçant un trait de peinture noire, recouvert ensuite avec le remplissage intérieur (fig. 3). Les còlés des grands carrés sont le support de lignes de points rougres : un gros point central souligné en haut et en bas de deux points plus petits. et de deux autres points de rhaque coté sur l'axe. Ies intersections du petit quadrillage oblique sont marquées de roix bleues qui sont remplacées par des points sur la premiere rangée suivant la bordure du décor (fig. 4 et 5):

La bordure qui devail encadrer ce molif grométrique est altestée sur au moins deux colés, perpendiculaires l'un à l'autre par le sens des tubulures du revers. C'est une bande verte, doublée a l'inlérieur par une bande rouge plus élroite, large au lolal de 6 a $8 \mathrm{~cm}$. Le quadrillage a été tracé sur l'ensemble de la surface a peindre. sans ménager l'emplacement de la bande d'encadrement. Celle-ci empiete donc naturellement sur la premiere rangée de carrés.

Sur certains fragments apparaît le dessin d'un grand cercle, superposé à la trame géométrique orthogonale (fig. 6). Son diamètre extérieur peut être évalué a $58 \mathrm{~cm}$. Il est délimité par une bande noire (5) $\mathrm{mm}$, bordée intérieurement el exlérieurement par un filet bleu (de 8 i $12 \mathrm{~mm}$ ). Le décor intérieur est malheu- 

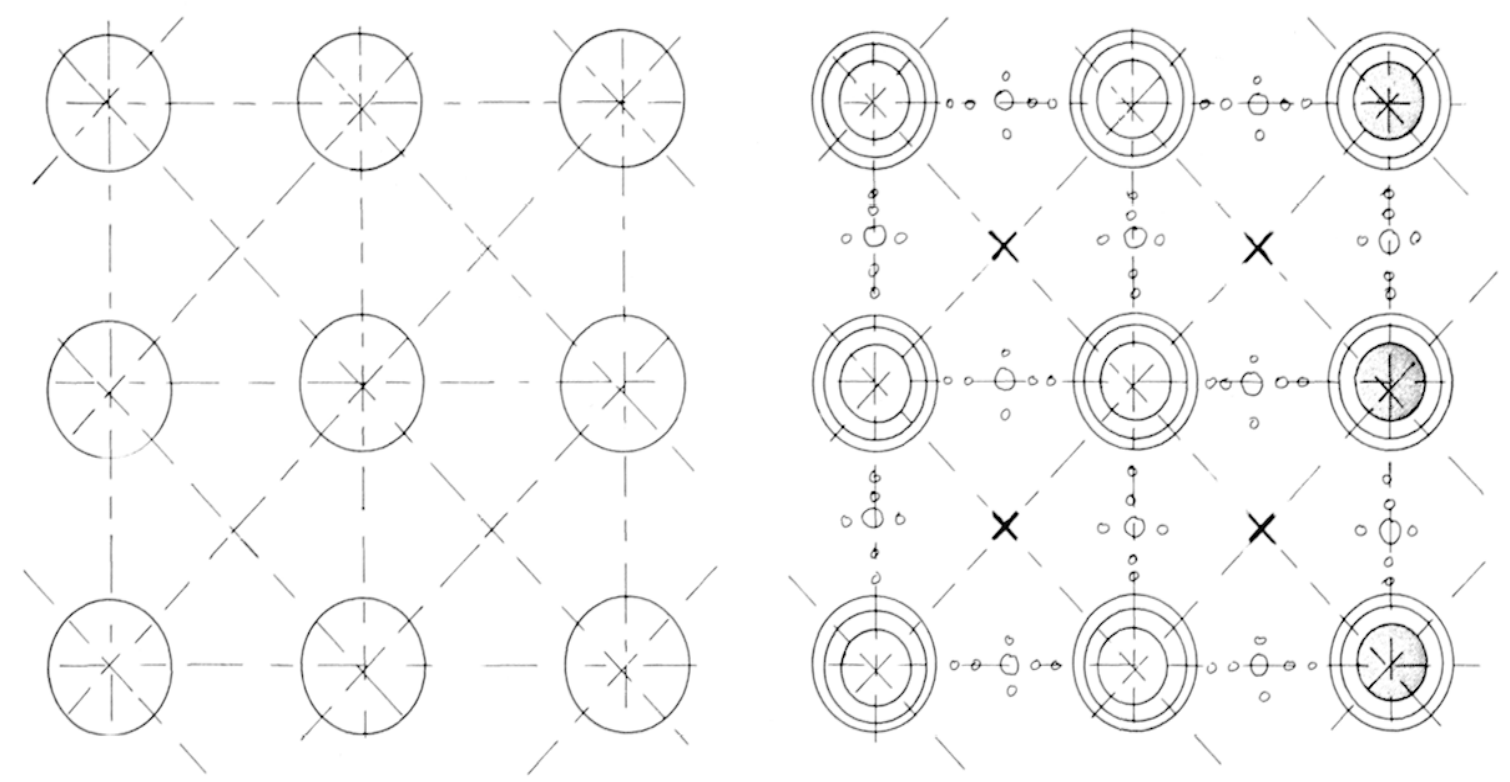

2 Construction géometrique du décor.

reusement indiscernable : toutefois, des restes de couleurs roses et brunes, soit en contact avec la bordure, soit sur des fragments isolés, évoquent quelque modelé. On peut supposer que ce cercle, conçu comme un médaillon central, contenait un motif beaucoup plus riche que la simple trame du fond el peut-être figuré (fig. 7 ).

linfin, un petit groupe de fragments, que l'on doit, par l'aspect du support et la gamme des couleurs employées, inclure dans le mème ensemble, porte un champ blanc uni, sans le décor répétitif habituel (fig. 8). Il est limité d'un còté par une bande rectiligne rouge (largeur 45 mm), bordant l'arête du mortier ; de l'autre par une bande courbe verte bordée de bleu (largeur totale $30 \mathrm{~mm}$ ), également arrêtée par la fin du mortier. La restitution donne done une forme triangulaire à un coité curviligne, qui pourrait par exemple provenir de l'écoinçon le long d'une ouverture arrondie. L'appartenance au mème ensemble est incontestable, mais aucun lien ne peut ètre donné avec le plafond proprement dit. Était-ce l'entrée de la niche, dont nous aurions ainsi un restige?

\section{Le deuxième état.}

Toute cette surface a été piquelée volontaire- ment, pour recevoir un nouvel enduit qui doit. pouvoir adhérer sur la surface sèche (fig. 6).

Cette couche postérieure de mortier subsiste par endroit : sable gris assez fin, lié à la chaux (de 5) mm d'épaisseur environ), recouvert d'l $\mathrm{mm}$ de chaux brossée el qui n'a apparemment pas été peinte. Le décor a simplement été recouvert, mais non remplacé. La raison qui a entraîné cette réfection n'est donc sans doute pas d'ordre esthétique, mais plutiot. fonctionnel. Il est banal que l'enduit des salles rhaudes ait besoin d'ètre rénové : si un souci d'économie y oblige, on passe une simple couche supplémentaire pour parer au plus pressé, sans chercher à refaire un décor. C'est. probablement ce qui s'est passé ici.

\section{Les plafonds à réseau régulier.}

Les sytèmes décoratifs à réseau régulier sont extrèmement fréquents, el bien caractéristiques des plafonds, bien qu'on ait pu, par exception. les employer sur des parois ${ }^{1}$. Sur

1 Quelques parois campaniennes ont en effet recu un tel décor en réseau à motif répétitif : citons la pièce à droite du tablinum dans la maison d'Ariane à Pompéi (VII, 4,51'; le comptoir d'une boutique Via dell' Abbondanza III, 2, 3, la chambre des Amours Iorés dans la maison sỵnonyme $(V I, 16,7$, et le décor 


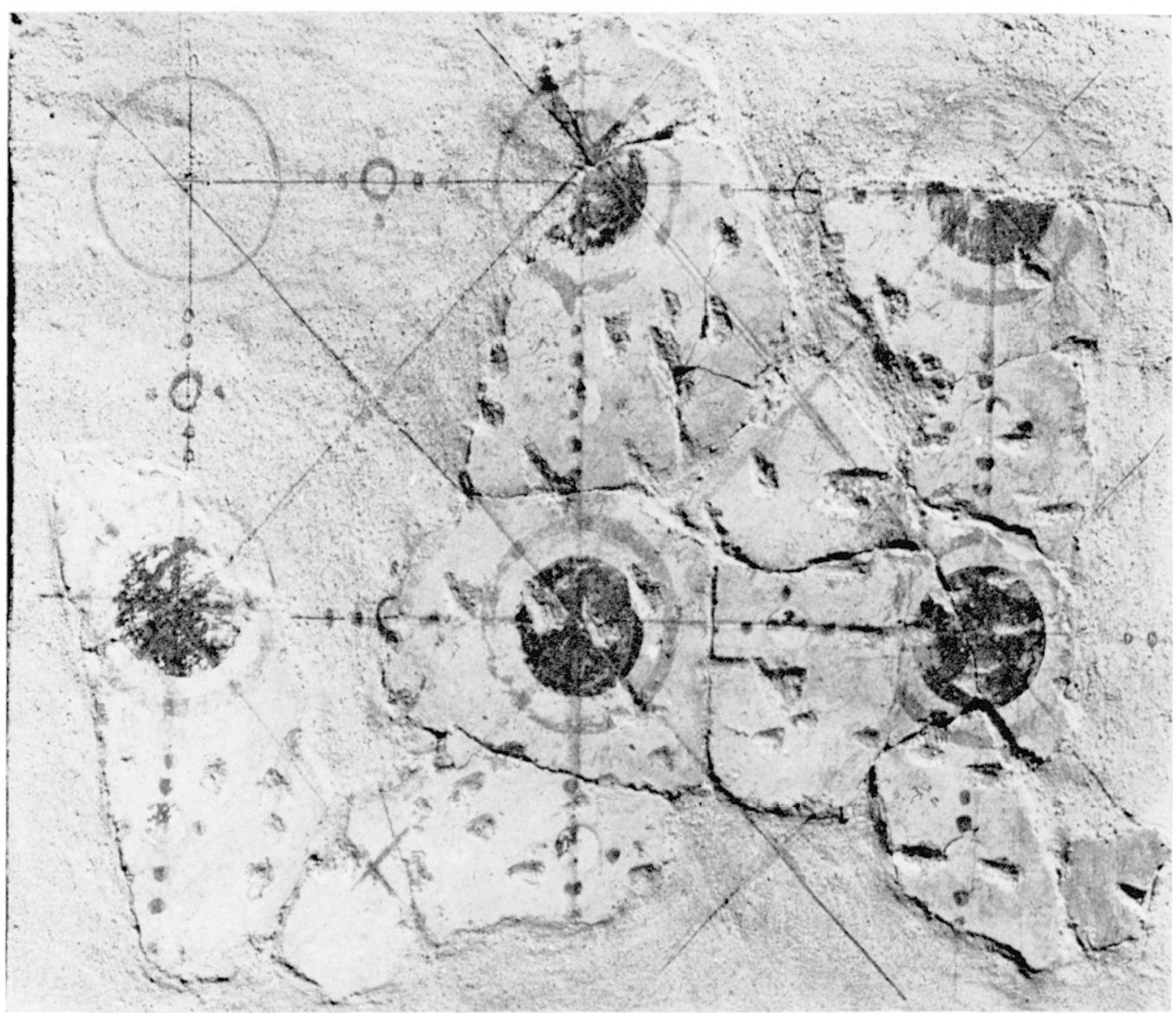

3 Échantillon de la peinture reconstitue.

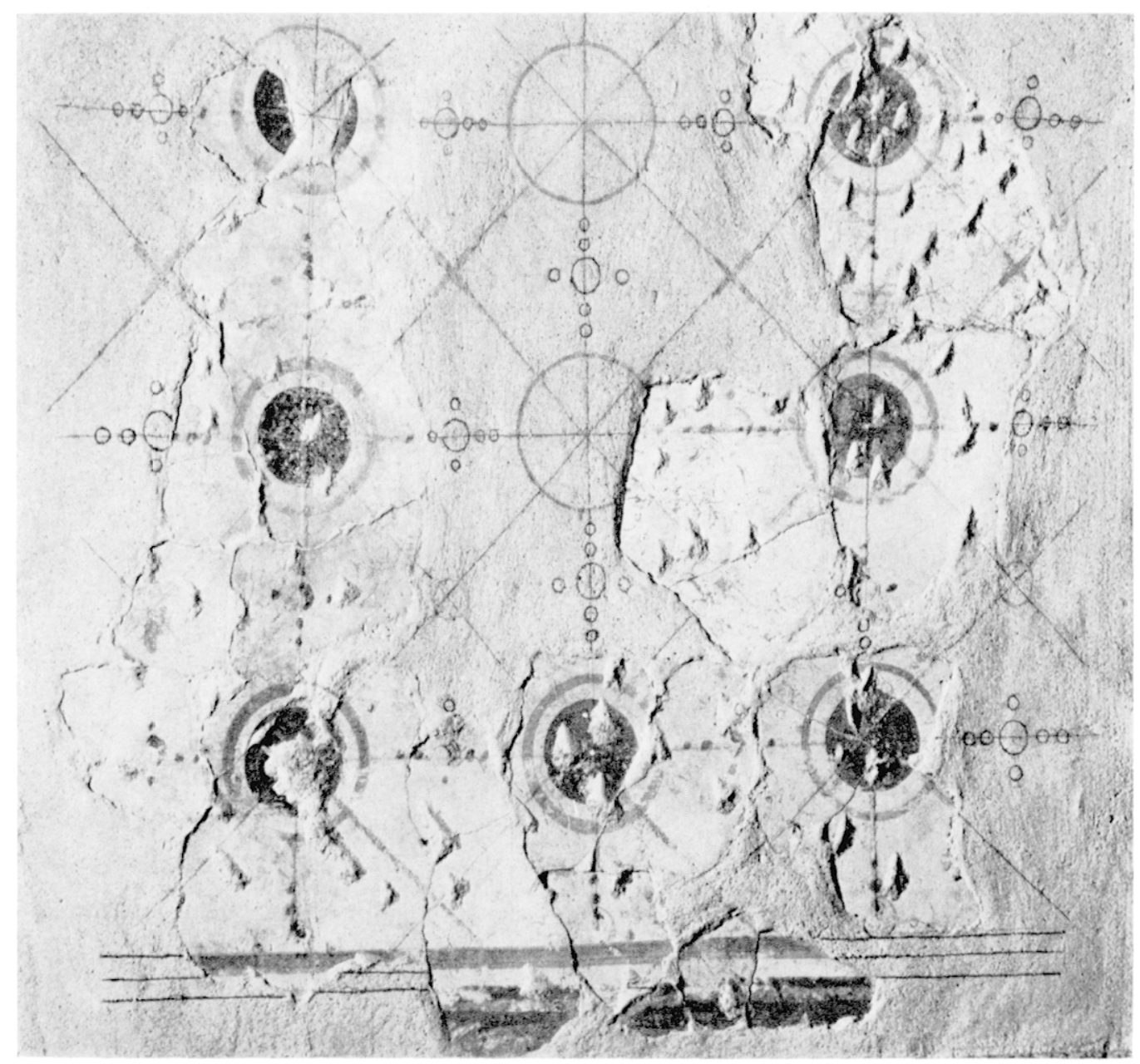

4 Échantillon de la peinture reconstiture, ave la hande d'encadrement. 


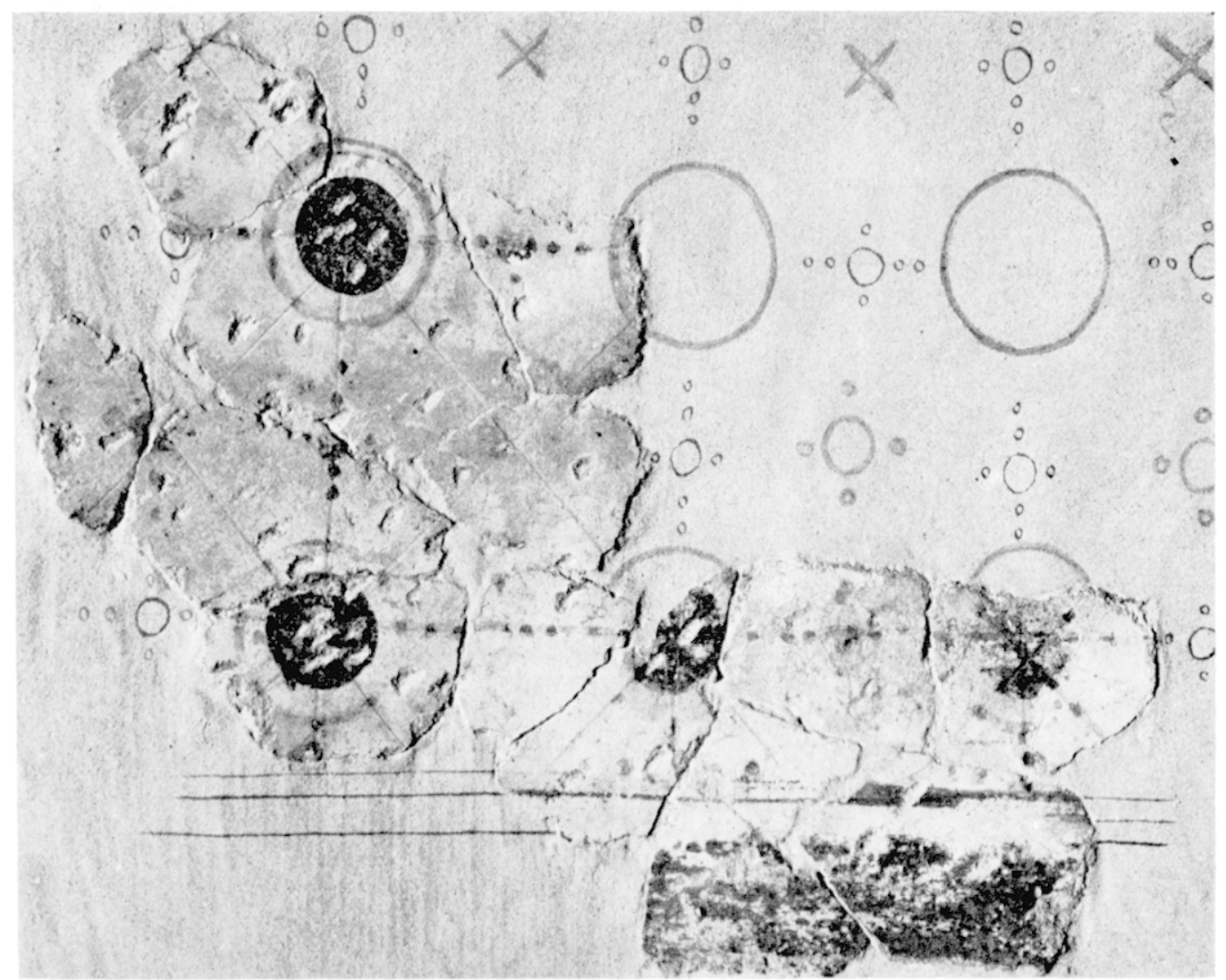

5) Échantillon de la peinture reconstituere, avec la bande dencadrement.

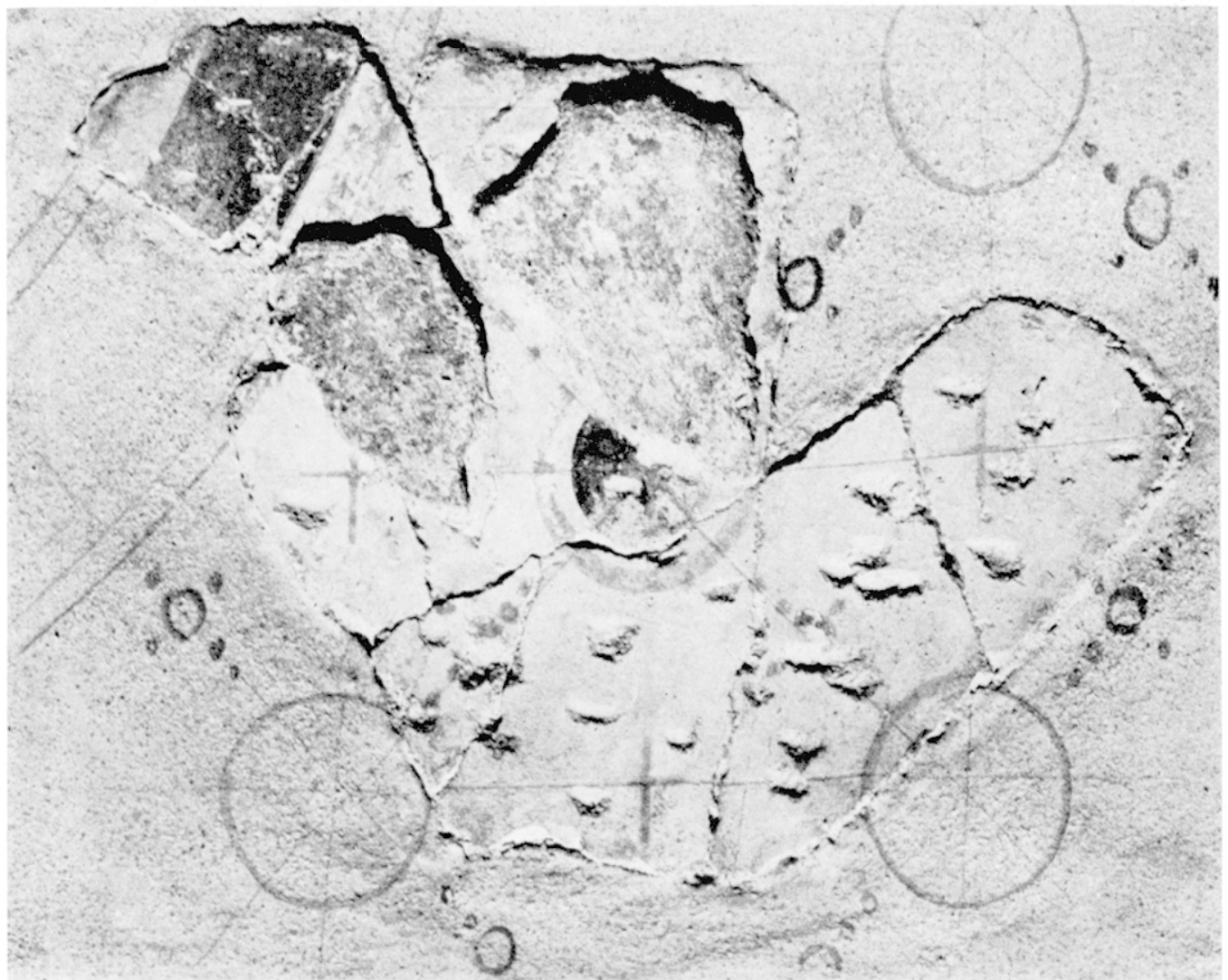

6 Échantillon de la peinture reconstiture, partie centrale, hordure du medaillon, et vestiges du deuxieme état. 


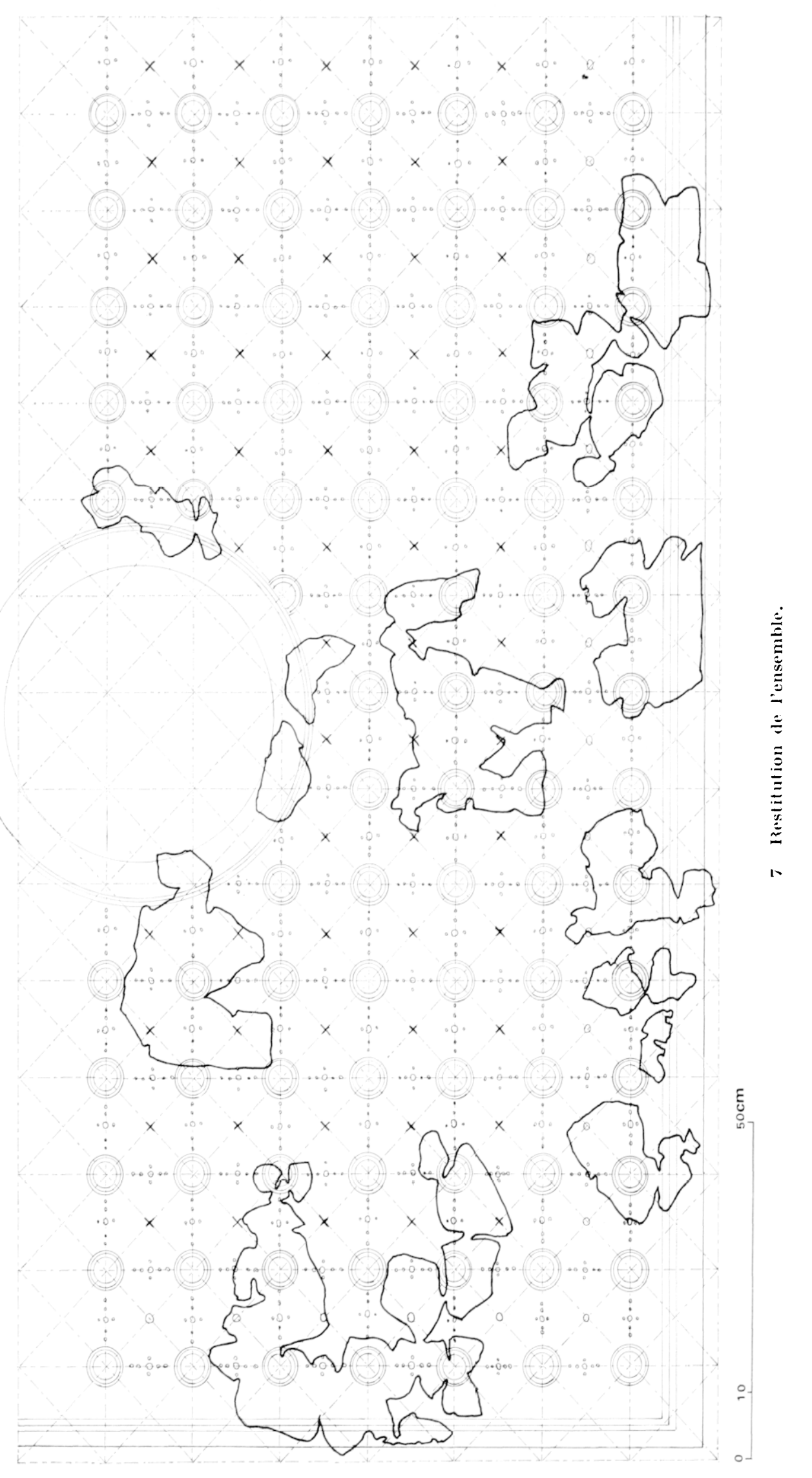




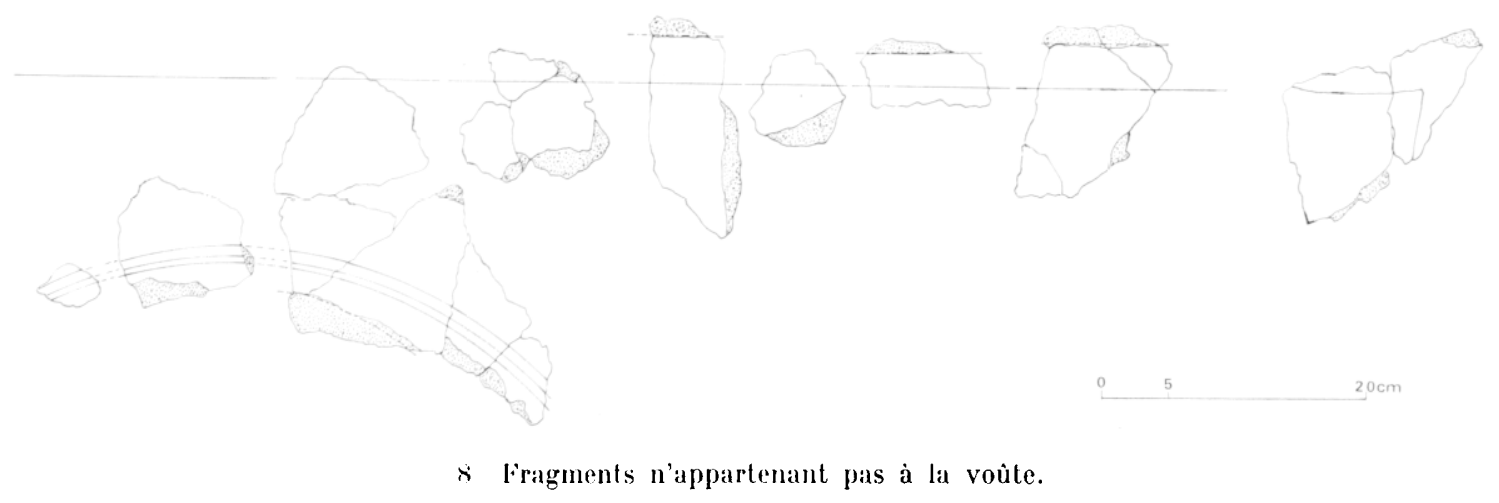

un schéma de base constant ..- un simple quadrillage - - et le principe d'un motif répété, les effets obtenus sont diversifies, selon le dessin choisi, par des variantes de détail.

Les exemples, très nombreux, attestent d'une infinité de motifs simples ou complexes, plus ou moins colorés et ornementés, mais tous comparables entre eux. Le plus simple, qui annonce celte longue série sans s'y inclure tout-à-fait, est un semis d'étoiles, disposées en quinconce sur la voùte de l'entrée des thermes du forum à Pompéi. Lne autre représentation de "ciel étoilé " est peinte avec plus de rigueur à la palestre d'Herculanum, chaque étoile étant inscrite avec soin dans les cercles esquissés aux intersections d'un quadrillage de base ${ }^{2}$.

La version la plus fréquente est une alternance de petits cercles et de motifs végétaux schématiques, soulignant le réseau de base. Il existe en suisse plusieurs ensembles peints de cette manière : ainsi, à Allaz, la restitution du plafond d'une villa ruslica offre une trame faite de guirlandes rigides, marquées d'un pelit, cercle aux intersections; dans chaque carré ainsi formé, est peint un médaillon encerclant.

beaucoup plus somptueusement ornemente d'un oecus à la Villa de Varano à Stabies.

2 I.e dessin grometrique de ces deux plafonds, ainsi que ceux des parois prealablement cities, ont étŕ étudiés dans : C. Ar.aAi, Esquisses el tracés préparaloires ans la peinlure marale campanienne, Ile partio

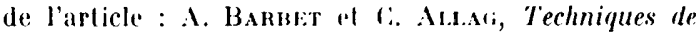
préparalion des parois dans la peinlure murale romaine, dans Melanges de l'Ecole frangaise de Rome, Inliquile (.M.E.F.R.1.), 8.1, 197:2, 2, p. 935-1069. un motif floral ${ }^{3}$. Ln décor, dont la description serait tout-à-fait identique, traité en ocre rouge, ocre jaune et marron, sur fond blane, ornait un corridor, à Avenches (fig. 9). A Martigny, sur une trame semblable, des filets aver des nouds de ruban schématiques dessinent les còtés des carrés, et les cercles intermédiaires sont remplis de polygones à côtés concaves, au lieu d'un fleuron ${ }^{5}$.

A Amiens également, nous trouvons un réseau de même type que celui d'Allaz ou d'Avenches, mais traité d'une manière schématique proche de celle d'Orléans. En eflet, les guirlandes de feuillages ne sont plus que des successions de taches vertes, informes pour la plupart. La bordure extérieure des grands cercles, rouge, est renforcée de gros points, rouges également; leur bordure intérieure est, noire. Les petits cercles sont remplis de rouge uni. Les fleurons, cruciformes, sont jaunes ${ }^{6}$ (fig. 10).

Par ailleurs, à còté de cet ensemble homogìne, on trouve de simples lignes de cercles concentriques, dont les cercles extérieurs tangents entre eux excluent les motifs intermédiaires et ne présentent donc pas le même aspect

3 W. Drack, Lie römische Wandmalereien der Schweiz, Bàle, 1950, en particulier, fig. 162.

4 W. Јнаск, Veu enldeclile römische Wandmalereien in der sichueiz, dans Antile Well, 1, 1981, p. 25, fig. 16 it 21 .

¿) Ibid., figr. ¿) (1) 15.

; J.-L. MAssy, Peinture murale gallo-romaine a Amiens, dans Revue du . Vord, tome 1,,$n^{0} 216$, janviormars 1976, p. 29-31, pl. VIJI-IX. 

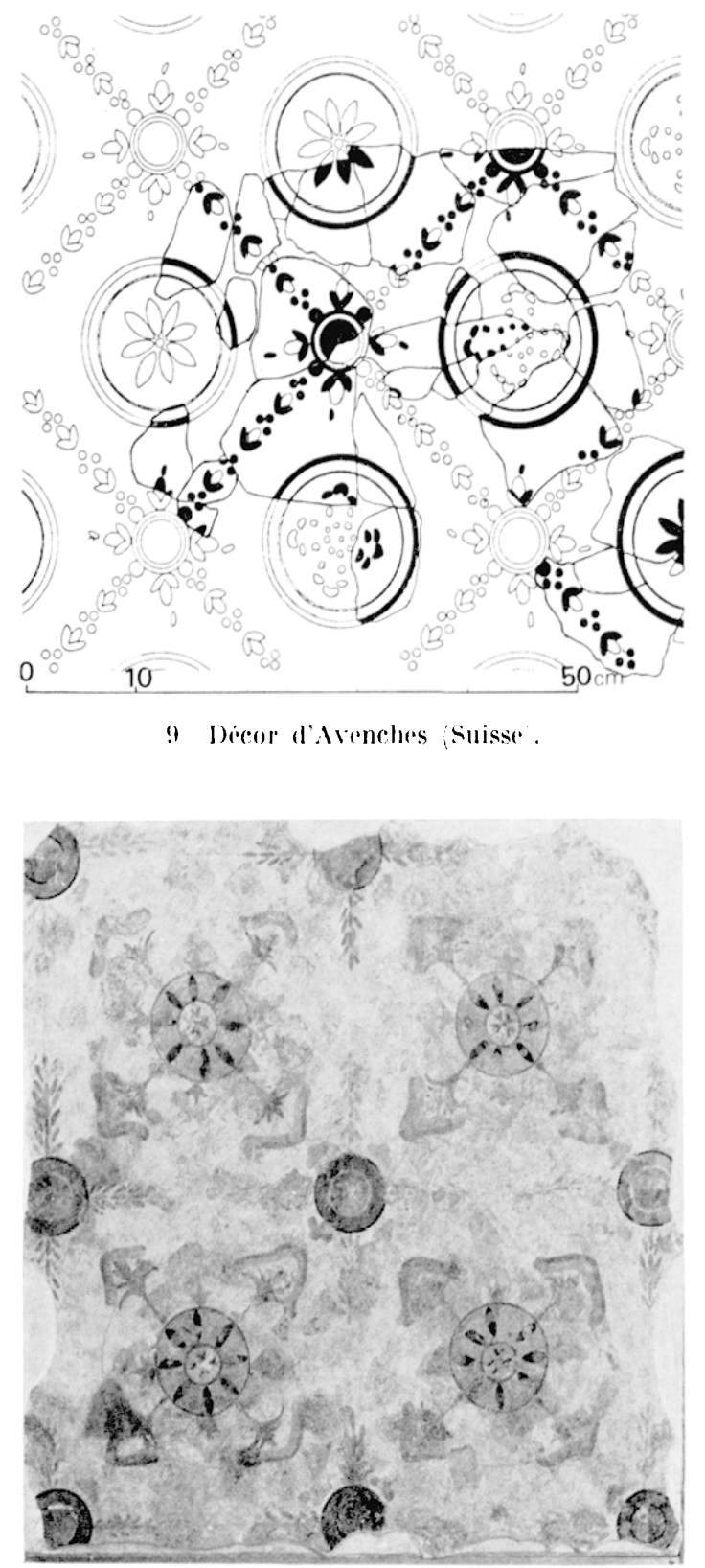

11 Plafond de Leicester.

final, comme a Chur . Trés différentes aussi par la richesse de l'ornementation, bien que fondées sur le mème principe. sont les peintures d'Holstein ${ }^{8}$, restituées selon toute vraisemblance en parlie supérieure de paroi, ou d'un autre ensemble d'Avenches" : dans res deux

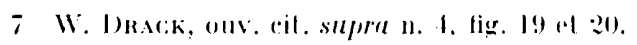

$\lambda$ Ibiol., fier. 4, $8 \times 11$.

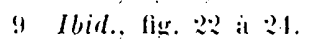

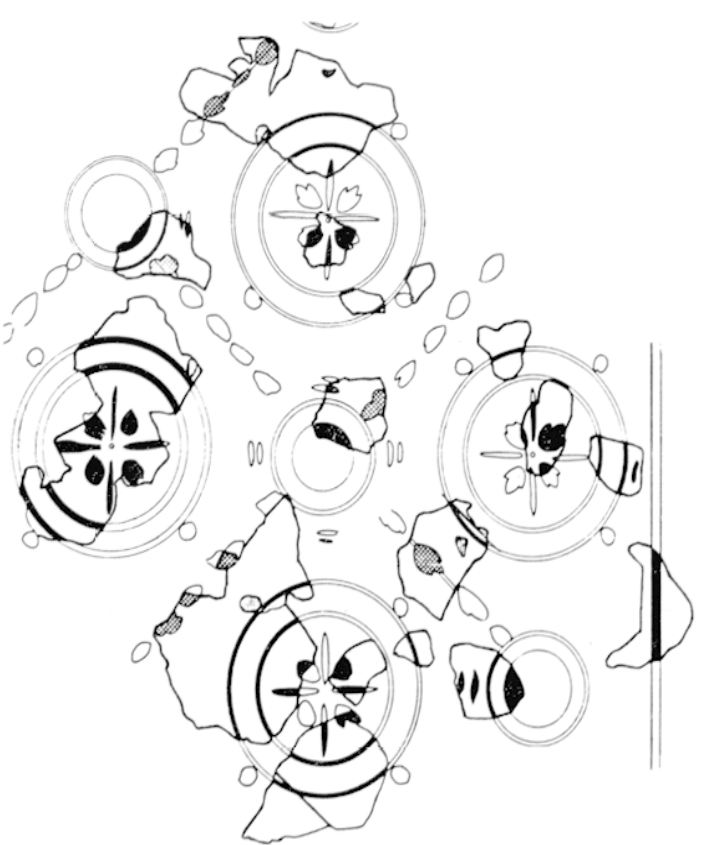

II) Lestilution de la peinture d'Amiens.

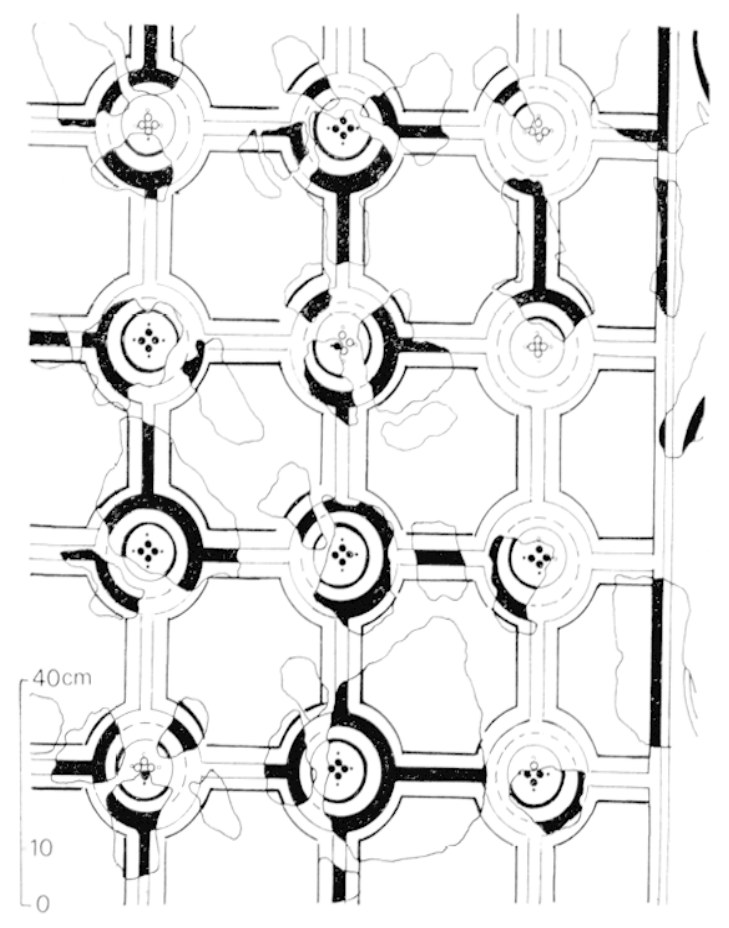

12.) Peinture dindilly.

(as, des rercles sécants se superposent à la trame orthogonale d'origine, el aboutissent a un reseau rurviligne complexe, que l'on peut lire soit comme des rosares juxlaposees, soit 
comme des carrés curvilignes reliés par les pointes ${ }^{10}$. Ce style paraît directement inspiré des décors stuqués et peints qui nous restent d'un Quatrième sityle pompéien en plein epanouissement : en particulier la voute des thermes de Stabies a Pompéi, el surtout la partie supérieure d'un mur disparu ; reproduil par Niccolini11. I)ans le cas d'Avenches (2e décor), le rapprochement aver le décor stuqué est d'autant plus immédiat que les contours sont peints en un "trompe l'oril" devant evoquer les petites frises ornementales d'oves et de languettes, moulures habituelles en sluc.

Cous retrouvons le schéma de cercles et de carrés en Grande-Brelagne, a I eicester en particulier ${ }^{12}$ (fig. 11) : les guirlandes schématiques dessinent les carrés, a l'intérieur desquels le dessin circulaire dont l'esquisse existe s'efface au profit d'un lleuron a quatre grands pétales el qualre plus petils. lin mème lype de fleuron inscrit dans un rercle existe a Carnunlum (Autriche), mais les motifs floraux $y$ sont traités plus souplement ${ }^{13}$. De mème, il existe a Karlsdorf-Thalerhof un quadrillage de guirlandes, avec de grands lleurons occupant, les espaces intermédiaires ${ }^{14}$.

A Collingham (Dalton Parlours) ${ }^{15}$, comme a Wroxeter ${ }^{16}$, de pelits arrés sur la pointe reliés entre eux par des bandes droites dessinent les octogones, oil sont inscrils des cercles. Des lleurons schématiques faits de simples points cun pour le corur. quatre pour les petales. soulignes de points plus pelits occupent le centre des carrés et des cerres. In plafond de

11) I.a premiere des restilutions proposenes correspond a la trame geomelrique la plus simple, ol pessemble a colle d'Halstein ; elle me semble la plus vaisemblable.

II F. Nacostix, Le case ed i monumenli di I'ompei disegnati e descrilli, Xaples, 1n54-18!6, II1, pl. XIV.

I:2 X. Daver, the conserualion of omano-British P'ainted Plaster, dans Brilannia, 3, 1972, p. 251 206x, fig. 11. Il s'agit du plafond d'un edifice public marche, a Blur Boar Iane, dale du debut du me siecle.

1:3 II. Bнaxnexstis, Wandmulereien aus Carnunlam, dans Cornunlum .Jahrbuch, Is5x, p. 10-19 jee partie.

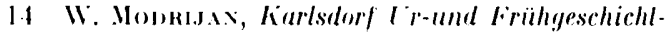
liche Funde, dans tichild emn . Sleier, Kleine .ichriften, $\therefore 1968$, 1. 3-111, fig. 1x-19.

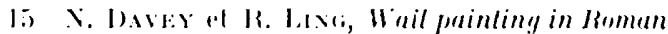

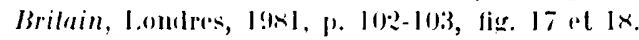

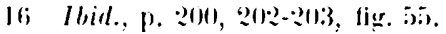

la rue de l'Abbé-de-l'Épée, à Paris ${ }^{17}$, est de conception identique ainsi qu'une peinture de Marzoll Lokr-Berchtesgaden ${ }^{18}$. malgré des différences minimes dans le traitement des fleurons.

A partir de ces interprétations moins souples oì prédominent les droites, les cercles peuvent disparaitre complètement, comme à Vet\% ${ }^{19}$ où ne subsistent que les octogones el les carrés. trame existant déjà en stuc au plafond des thermes de stabies par exemple ${ }^{20}$. En revanche. les rercles seuls peuvent etre privilégiés, comme a Andilly fix. 12) où, reliés entre eux par des bandes de mime rouleur frouge!. ils portent en leur centre de petits fleurons faits de quatre points. Les octogones intermédiaires ont qualre cotes concaves, sur les faces avoisinant les bordures externes des cercles ${ }^{21}$. In dessin analogue est utilise sur la voûte du lombeau de silistra (Bulgarie), mais des figures variés (hommes, obseaux. fleurs) enrichissent l'intérieur des octogones ${ }^{22}$.

Nolons enfin que le quadrillage se trouve parfois réduit à sa plus simple expression. l'exemple le plus précoce se trouve à Rome, dans un Colombarium, via Taranto : toule la surface de la voute est cloisonnée réguliprement par de fines quirlandes droites. Une rose ef deux feuilles, traitées en motif oblique alternativement à droite et à gauche, figurent au centre de chaque compartiment. I Wilcombe, en Grande-Brelagne 23 , le mème rompartimenlage par des tiges feuillues, agrementées de peliles baies. apparail : un rercle a fleuron central, de nouveau, alterne aver un petil

17 H. Hustos, Les enduils peints diponque gallu-

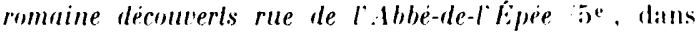
Cahiers de la Rolonde, 2, 197x, p. 11-:?!.

Is R. linnstasix, Ein römische riebïnde in Murzoll, dans Buyerisehe loryeschichls Bläle", $2 x$, 1969, p. $301-57$.

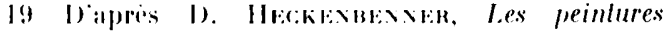
murales dip'sque gallo romaine en Lorraine. mimoire de maitrise, inedit, facalle des lettres de Naney, $197 \%$.

20) Voinle de l'apudiglerium.

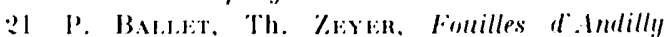
19;8-z!, dans Rerne archeolongique, siles, I!nul.

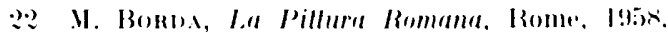
fier. de la l. 139 .

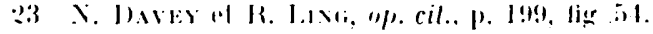


motif figuré (trophée?), au centre des carrés ainsi délimités. Le quadrillage de la partie supérieure du mur, dans la maison de Pinarius Cerealis à Pompéi, bien que sans aucune inspiration florale, relève du même principe; rosaces, monstre;, ou tètes mythologiques occupent chaque carré. Là encore, la similitude avec les plafonds stuqués n'est pas négligeable : les caissons, directement inspirés de l'architecture, sont traités avec plus de fantaisie lorsque l'art du placage le permet; les plafonds de la maison du Cryptoportique sont divisés en secteurs, dont chacun porte un réseau de carrés, de losanges ou d'octogones, garnis de fleurons, de trophéez ou d'objets décoratifs divers.

La petite voùte d'Orléans entre donc dans une catégorie bien définie. Son réseau, parallèle au sens de la bordure, appartient à la dernière série (Rome, Via Taranto, Witcombe), alors que la plupart des autres décors se lisent obliquement par rapport aux limites des parois ou du plafond. Wais ici, l'accent est mis sur les intersections que marquent les cercles colorés, el qui sont l'élément le plus visible de l'ensemble, comme sur les décors d'Amiens ou de suisse. Les coités des carrés ne sont indiqués que par des lignes de points rouges, qui remplacent les tiges végétales attendues -- de même qu'à Wroxeter ou a Andilly les points remplacent les fleurons. C'est donc une version schématique du motif végétal, qui ne doit pas nous étonner. Quant à la croix centrale, elle remplace sans doute l'ornement intérieur du caisson, réduil à sa plus simple expression.

On ne peut tirer de cet ensemble aucune conclusion stylistique ou chronologique. C'est un décor banal dans tout l'Empire Romain, et utilisé en tous lemps. Mais il est intéressant d'en voir, à partir d'un exemple, les différentes adaptations.

Claudine Allag

(G...R.R.S.)

N.B. - Les photographies at les dessins sont dus a $\mathrm{U}_{\text {mes }}$ Th. Adam, fig. 1, 2, 7 et 8 ; A. Barbet, fig. 3 ; H. Eristov, fig. 11. l.es fig. 4, 5 et 6 sont de l'auteur. Fig. 9, daprès W. DRAck, Neu entdechle römische Wandmalereien in der Schweiz, dans Antike Well, 1, 1981 , fig. 21 ; fig. 10, d’apris J.-L. Massy, Peinture murale gallo-romaine à Amiens, dans Revue du Nord, IV, no 216, 1976, fig. IX; fig. 12, d'apris P. BatLit, Th. Zayse, Fouilles d'Andilly 1978-1979, dans Revue archéslogique, siles, 1980. 